SUBJECT AREAS:

PARASITE BIOLOGY

MALARIA

DRUG DEVELOPMENT

Received

27 December 2013

Accepted

1 April 2014

Published

17 April 2014

Correspondence and requests for materials should be addressed to K.K. (kkato@obihiro. ac.jp) \section{falciparum growth and invasion of red blood cells in vitro}

Frances Cagayat Recuenco ${ }^{1,2}$, Kyousuke Kobayashi ${ }^{3}$, Akiko Ishiwa' ', Yukiko Enomoto-Rogers ${ }^{4}$, Noreen Grace V. Fundador ${ }^{4}$, Tatsuki Sugi 1,2, Hitoshi Takemae ${ }^{1,2}$, Tatsuya Iwanaga', Fumi Murakoshi', Haiyan Gong' ', Atsuko Inomata', Taisuke Horimoto', Tadahisa Iwata ${ }^{4} \&$ Kentaro Kato ${ }^{1,2}$

'Department of Veterinary Microbiology, Graduate School of Agricultural and Life Sciences, The University of Tokyo, 1-1-1 Yayoi, Bunkyo-ku, Tokyo 113-8657, Japan, ${ }^{2}$ National Research Center for Protozoan Diseases, Obihiro University of Agriculture and Veterinary Medicine, Inada-cho, Obihiro, Hokkaido 080-8555, Japan, ${ }^{3}$ Department of Host-Parasite Interaction, Division of Microbiology and Immunology, Institute of Medical Science, The University of Tokyo, 4-6-1 Shirokanedai, Minato-ku, Tokyo 1088639, Japan, ${ }^{4}$ Laboratory of Science of Polymeric Materials, Department of Biomaterial Sciences, Graduate School of Agricultural and Life Sciences, The University of Tokyo, 1-1-1 Yayoi, Bunkyo-ku, Tokyo 1 13-8657, Japan.

Here, we assessed the sulfated derivative of the microbial polysaccharide gellan gum and derivatives of $\lambda$ and к-carrageenans for their ability to inhibit Plasmodium falciparum 3D7 and Dd2 growth and invasion of red blood cells in vitro. Growth inhibition was assessed by means of flow cytometry after a 96-h exposure to the inhibitors and invasion inhibition was assessed by counting ring parasites after a 20-h exposure to them. Gellan sulfate strongly inhibited invasion and modestly inhibited growth for both $P$. falciparum 3D7 and Dd2; both inhibitory effects exceeded those achieved with native gellan gum. The hydrolyzed $\lambda$-carrageenan and oversulfated $\kappa$-carrageenan were less inhibitory than their native forms. In vitro cytotoxicity and anticoagulation assays performed to determine the suitability of the modified polysaccharides for in vivo studies showed that our synthesized gellan sulfate had low cytotoxicity and anticoagulant activity.

$\mathrm{T}$ he estimated 660,000 deaths from 220 million cases of malaria reported by the WHO in $2010^{1}$ represents a decline in the mortality caused by malaria especially in the sub-Saharan endemic areas where most cases occur $^{2}$. This success can be attributed to programs that include distribution of insecticide-treated nets, use of indoor residual spraying, and expansion of malarial rapid diagnostic tests. Nevertheless, because of the constant threat of the Plasmodium parasites and the Anopheles vectors developing resistance to established antimalarials and insecticides, the drive to develop alternative antimalarial drugs, insecticides, and improved rapid diagnostic tests continues ${ }^{2}$.

Plasmodium parasites, the causative agent of malaria, are obligate intracellular protozoa transmitted from the blood meal of the female Anopheles mosquito to humans. In the erythrocytic stage of the disease, the Plasmodium merozoites invade red blood cells, which results in their destruction and the release of the parasite and erythrocytic material into the circulation. The host response to these events manifests into the clinical symptoms of the disease, which include intermittent fever, abdominal pain, anemia, and general weakness ${ }^{3}$.

Successful invasion of the erythrocyte is crucial for the survival of the malaria parasite. Upon egress, there is a small window of time when the free malaria merozoites are exposed to the host's immune system, which provides an opportunity to target the parasites with vaccines or drugs ${ }^{4}$.

Erythrocyte invasion by the Plasmodium merozoite is a complex, multi-step process that involves interactions between the parasite and host cell proteins. The initial reversible attachment of the merozoite to the red blood cell may involve proteins on the merozoite surface, although evidence to support this concept is lacking. During red cell binding, the merozoite reorients so that the apical complex makes contact with the erythrocyte surface. Here, the micronemes secrete invasion proteins, such as apical membrane antigen 1 (AMA1) and erythrocyte bindinglike proteins (EBLs), and the rhoptries secrete reticulocyte binding-like proteins (RBLs) and rhoptry neck proteins (RONs). AMA1, a major candidate for a multicomponent vaccine against malaria ${ }^{5}$, interacts with the rhoptry neck proteins RON2, RON4, and RON5 to form a complex that is a critical component of the moving junction. The EBLs and RBLs bind with receptors on the red blood cell and are implicated in host cell selection and 
alternative invasion pathways for the Plasmodium merozoite. Merozoite entry into the red blood cell proceeds as the merozoite is propelled by an actin-myosin motor complex, with simultaneous shedding of the surface proteins to enable the parasite to adapt to its new intracellular environment ${ }^{6-8}$.

Sulfated glycosaminoglycans (GAGs), such as heparin ${ }^{9}$, dextran sulfate, fucoidan ${ }^{10,11}$, and fucosylated chondroitin sulfate ${ }^{12}$ have been shown to inhibit merozoite entry into erythrocytes in vitro. In addition, sulfated GAGs have been shown to prevent the cytoadherence of Plasmodium-infected red blood cells in vitro ${ }^{10-15}$.

Heparin, in particular, appears to target several merozoite proteins ${ }^{16,17}$, including invasion proteins such as merozoite surface protein 1 (MSP1) ${ }^{18}$ and erythrocyte binding antigen 140 (EBA-140) ${ }^{19}$. Thus, unlike other antimalarials, heparin can inhibit the invasion of red blood cells by the Plasmodium merozoites ${ }^{20}$. MSP1 is a major candidate for a multi-component malaria vaccine ${ }^{5}$, and EBA-140, also known as BAEBL, is an erythrocyte binding ligand that interacts with the glycophorin $C$ receptor on the erythrocyte surface ${ }^{21}$. These findings provide clues as to how these invasion proteins interact with cell surface proteoglycans, such as heparan sulfate, and could help explain the mechanism by which sulfated polysaccharides inhibit parasite entry into red blood cells. However, because heparin is a potent anticoagulant, it cannot be used to treat clinical malaria ${ }^{22}$.

Sulfated polysaccharides from marine sources are currently being exploited for their potential therapeutic applications. These include carrageenans from seaweeds, of which there are three major types: kappa $(\kappa)$, lambda $(\lambda)$, and iota ( $(1)$. These carrageenans differ in their levels of sulfation, which may also account for their different gelling properties. The carrageenans inhibit P. falciparum 3D7 and Dd2 growth and invasion of red blood cells in vitro ${ }^{23}$. In vivo, $\mathrm{A} / \mathrm{J}$ mice pre-treated with calcium carrageenan prior to infection with $P$. berghei NK65A showed lower parasitemia compared with the untreated group but this pre-treatment did not promote recovery or survival of the animals ${ }^{24}$. In addition, Huber et al. ${ }^{25}$ showed that there was an increase in the permeability of the blood brain barrier in rats $72 \mathrm{~h}$ after subcutaneous administration of $\lambda$-carrageenan. This side effect could enhance the development of cerebral malaria and is thus a major disadvantage of carrageenans for therapeutic use in malaria.

Modification of $\kappa$-carrageenan improved its potential for use against various pathogens. The basic structure of $\kappa$-Carrageenan consists of $(1 \rightarrow 3)$ - $\beta$-D-galactose (G unit) - ( $1 \rightarrow 4)$ - $\alpha$ - 3,6-anhydro $\mathrm{D}$-galactose (A unit). Acetylated, sulfated, and phosphorylated $\kappa$ carrageenan derivatives show enhanced antioxidant and antitumor properties $^{26}$. Other $\kappa$-carrageenan derivatives have shown activity against tumor cells ${ }^{27,28}$, bacteria ${ }^{29}$ and viruses, including influenza virus $^{30,31}$, and human immunodeficiency virus ${ }^{32}$. We, therefore, sought to determine whether modification of $\kappa$-carrageenan and $\lambda$-carrageenan could affect their action against malaria parasites and their safety as potential adjunct therapy for malaria.

Gellan gum (GG) is a linear, anionic, high molecular weight, microbial polysaccharide produced by the bacterium Sphingomonas (Pseudomonas) elodea (ATCC 31461). It is a thermoreversible gel, noted for its high gel strength and stability, which make it useful as a drug vehicle, food additive, component of personal care products, and microbiological media. Its basic repeating unit is a tetrasaccharide, consisting of two glucose (Glc) residues, one glucuronic acid (GlcA), and one rhamnose (Rha) residue: $[\rightarrow 3)-\beta-\mathrm{D}-\mathrm{Gl} c p-(1 \rightarrow$ $4)-\beta$-D-GlcpA- $(1 \rightarrow 4)-\beta$-D-Glcp- $(1 \rightarrow 4)-\alpha$-L-Rhap- $(1 \rightarrow]^{33-36}$. Animals studies involving gellan gum addition to the diet or being given by gavage have shown it to be inert and safe for internal use $\mathrm{u}^{35}$. In vivo, GG has been found to have low induction of inflammation, so GG-based materials have been evaluated for potential therapeutic applications that include films to reduce post-surgical adhesions ${ }^{37-39}$ and discs for nucleus pulposus regeneration ${ }^{40}$. GG-based hydrogels were also found to support viability of encapsulated chondrocytes intended for cartilage transplants ${ }^{41}$.
Gellan gum has been modified to obtain sulfated derivatives (gellan sulfate $)^{42}$ that have anticoagulant activities similar to those of heparin, with the exception of one derivative (GS1), which had the lowest sulfonation ratio (5.0\%), and the least anticoagulant activity ${ }^{43}$. The in vivo effects of gellan sulfate, however, appear not to have been fully explored yet. To our knowledge, there are no published reports on the in vitro effects of gellan gum and gellan sulfate on malaria parasites.

The goal of this study was to assess synthetic compounds that inhibit merozoite entry into red blood cells, similarly to heparin, but with a better safety profile. We evaluated the microbial polysaccharide gellan gum and prepared its sulfated derivative, gellan sulfate, and the carrageenan derivatives, hydrolyzed $\lambda$-carrageenan and oversulfated $\kappa$-carrageenan, for their inhibitory effects on Plasmodium falciparum 3D7 and Dd2 growth and invasion of erythrocytes. We also tested these compounds for their cytotoxicity to $293 \mathrm{~T}$ cells. In addition, we assessed the in vitro anticoagulant activity of our synthesized gellan sulfate for its suitability for in vivo studies.

\section{Results}

Synthesis of gellan gum, $\lambda$ - and $\kappa$-carrageenan derivatives. $\kappa$ Carrageenan and gellan gum were sulfated by the $\mathrm{SO}_{3}$-pyridine complex. The $\kappa$-carrageenan and gellan sulfates, and the hydrolyzed $\lambda$-carrageenan were more readily soluble in distilled water at room temperature compared with their native counterparts. The modified polysaccharides also did not form gels in solution.

The degree of substitution (DS) of the sulfonyl group and sulfation rate (\%) were determined by elemental analyses and are listed in Table 1 . The DSs for $\kappa$-carrageenan and gellan were defined as the molar number of sulfonyl group per di-saccharide and tetra-saccharide unit, respectively.

NMR analyses were used to confirm the sulfation of $\kappa$-carrageenan and gellan. ${ }^{13} \mathrm{C}$-NMR spectra before and after sulfation are available in the Supplemental data (Supplementary Figs. S1 and S2). The peaks of native $\kappa$-carrageenan and gellan were assigned according to the assignments reported previously ${ }^{44-46}$. The major peaks of sulfated $\kappa$-carrageenan with a DS of 3.0 were also assigned according to the assignments for sulfated $\kappa$-carrageenan with a DS of 3.1 reported elsewhere $e^{47,48}$. The overlapped peaks of ring-carbons of gellan sulfate with a DS of 3.7 were rarely assigned due to their complex appearance caused by sulfonyl substituents and remaining hydroxyl groups. In the case of $\kappa$-carrageenan, shifts of the peaks of C6 (G) from 61.4 to 66.5 ppm, C2 (G) from 69.7 to $75.4 \mathrm{ppm}$ and C2 (A) from 70.0 to $72.7 \mathrm{ppm}$ indicated successful sulfation at these positions. The complete disappearance of the peak assigned C6 $(\mathrm{G})$ indicated that the C6 (G) position was completely substituted. Other peaks were likely assigned to ring carbons of un-substituted sugar units. In the case of gellan, the peaks assigned to C6 (13 G) at $61.7 \mathrm{ppm}, \mathrm{C6}(14 \mathrm{G})$ at $62.2 \mathrm{ppm}, \mathrm{C} 2(\mathrm{R})$ at $71.6 \mathrm{ppm}$, and $\mathrm{C} 3(\mathrm{R})$ at $71.7 \mathrm{ppm}$, completely disappeared, indicating preferential sulfation at these positions.

\begin{tabular}{|c|c|c|c|c|c|}
\hline \multirow[b]{2}{*}{ Sulfated derivatives } & \multicolumn{3}{|c|}{ Elemental analysis (\%) } & \multirow[b]{2}{*}{$\mathrm{DS}^{a}$} & \multirow{2}{*}{$\begin{array}{l}\text { Sulfation rate } \\
(\%)^{a}\end{array}$} \\
\hline & C & $\mathrm{H}$ & $S$ & & \\
\hline $\begin{array}{l}\text { к-carrageenan } \\
\text { Gellan }\end{array}$ & $\begin{array}{l}20.3 \\
23.3\end{array}$ & $\begin{array}{l}4.09 \\
4.38\end{array}$ & $\begin{array}{l}13.6 \\
9.57\end{array}$ & $\begin{array}{l}3.0 \\
3.7\end{array}$ & $\begin{array}{l}75 \\
37\end{array}$ \\
\hline \multicolumn{6}{|c|}{$\begin{array}{l}\text { oRelative to maximun DS: } 4.0 \text { ( } \mathrm{k} \text {-carrageenan), } 10.0 \text { (gellan). } \\
\text { DS - degree of substitution. } \\
\text { Percent carbon (C), hydrogen (H), and sulfur (S) as determined by elemental analyses. DS was } \\
\text { calculated according to the equation of Rochas, etal.45, that is, DS }=(S \% / \text { atomic mass of S)/(C\%/ } / \text { / } \\
\text { atomic mass of } C \times \text { number of carbons for one unit). Sulfation rate }(\%) \text { was calculated by taking the } \\
\text { ratio of the DS of the sulfonyl group to the maximum DS of } 4.0 \text { for } \kappa \text {-carrageenan and } 10.0 \text { for } \\
\text { gellan. }\end{array}$} \\
\hline
\end{tabular}




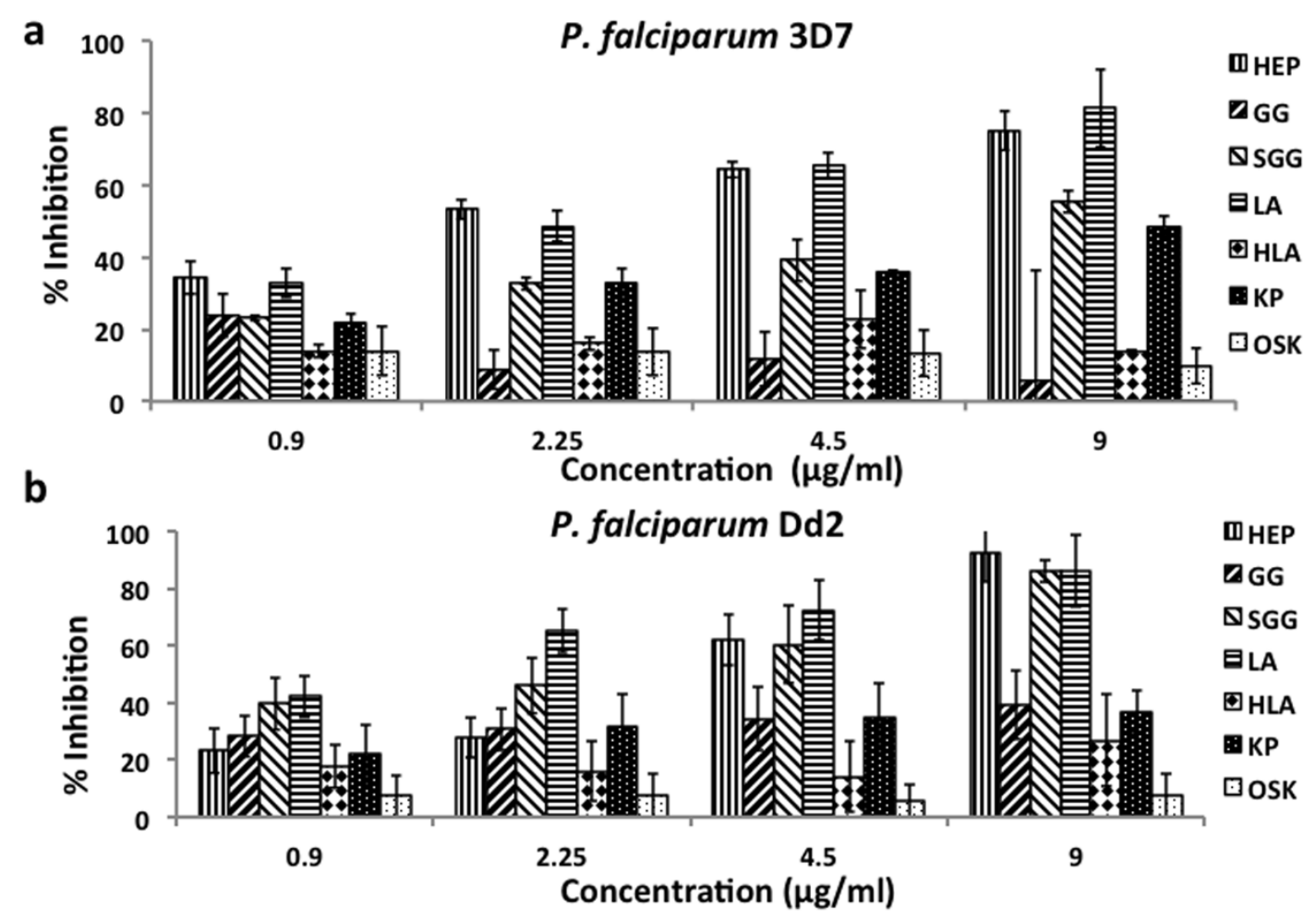

Figure $1 \mid$ In vitro growth inhibition of $\boldsymbol{P}$. falciparum 3D7 (A) and Dd2 (B). Late stage P. falciparum 3D7 and Dd2 trophozoites (parasitemia $0.3 \%$, hematocrit $1 \%$ ) were cultured in the presence of the following inhibitors: heparin (HEP), gellan gum (GG), gellan sulfate (SGG), $\lambda$-carrageenan (LA), hydrolyzed $\lambda$-carrageenan (HLA), $\kappa$-carrageenan (KP), and oversulfated $\kappa$-carrageenan (OSK) in 96 -well round-bottom plates for 96 h. Final parasitemia was determined by use of flow cytometry. Percent inhibition is shown ( $\mathrm{y}$-axis) against increasing concentrations (x-axis) of each inhibitor. At $9 \mu \mathrm{g} / \mathrm{ml}$, SGG inhibited the growth of $P$. falciparum 3D7 by $55.33 \%$ and that of Dd2 by $85.83 \%$. At the same concentration, GG, HLA, and OSK inhibited both P. falciparum lines by $20 \%$ or less. Error bars represent standard deviations from the means of triplicate assays from three independent experiments.

Growth inhibition assay. The native $\lambda$ - and $\kappa$-carrageenans inhibited the growth of P. falciparum 3D7 (Figure 1A) and Dd2 (Figure $1 \mathrm{~B})$ in vitro in a concentration-dependent manner consistent with the observations of Adams et al. ${ }^{23}$. Both the hydrolyzed $\lambda$ carrageenan and the oversulfated $\kappa$-carrageenan derivatives poorly inhibited P. falciparum 3D7 and Dd2 growth in vitro. Gellan sulfate $(9 \mu \mathrm{g} / \mathrm{ml})$ inhibited growth of P. falciparum 3D7 (55.33\%) and Dd2 $(85.83 \%)$ in vitro (Figure $1 \mathrm{~A}$ and $1 \mathrm{~B})$. In contrast, native gellan gum exhibited low inhibition $(5.58 \%, 3 \mathrm{D} 7$; and, $39.22 \%, \mathrm{Dd} 2)$ at the same concentration. We, therefore, chose to assess gellan sulfate and the native gellan gum for their ability to inhibit invasion.

Invasion inhibition assay. At $10 \mu \mathrm{g} / \mathrm{ml}$, gellan sulfate showed strong inhibition of invasion by $P$. falciparum 3D7 (93.92\%) and Dd2 $(88.39 \%)$ in vitro (Figure 2). Heparin also showed inhibition of invasion by $P$. falciparum 3D7 (69\%) and Dd2 (89.03\%). Native gellan gum showed moderate inhibition of invasion for both $P$. falciparum 3D7 and Dd2 (46.13\% and 47.51\%).

Cytotoxicity assays. Unlike native gellan gum, gellan sulfate does not gel in solution. With this change in property, we wanted to determine the safety of gellan sulfate for future in vivo studies. Hence, we performed MTT cytotoxicity assays on 293T cells using the original selection of inhibitors for comparison (Figure 3A). Heparin and native gellan gum treatment caused a marked decrease in cell viability at concentrations of 250 and $500 \mu \mathrm{g} / \mathrm{ml}(54.68 \%$ and $70.18 \%$ for heparin; $79.86 \%$ and $57.74 \%$ for gellan gum). By contrast, gellan sulfate, as well as the native $\lambda$ - and $\kappa$ - carrageenans and their respective derivatives, showed relatively low cytotoxicity at $500 \mu \mathrm{g} / \mathrm{ml}$ (86.44\%, 81.52\%, 89.33\%, $101.88 \%$, and $94.30 \%$, respectively).
In vitro anticoagulant activity of gellan sulfate. The in vitro anticoagulant activity of the gellan sulfate was assessed by using activated partial thromboplastin time (APTT) anticoagulant assays with healthy mouse plasma ${ }^{49}$. In separate experiments, the APTT values for normal plasma from a healthy animal ranged from $16.0 \mathrm{~s}$ to $27.1 \mathrm{~s}$, with a mean value of $23.4 \mathrm{~s}$. The APTT for plasma with heparin at 10 and $100 \mu \mathrm{g} / \mathrm{ml}$ increased dramatically (mean $=$ $181.0 \mathrm{~s}$ ), whereas mean APTTs for plasma with 10 and $100 \mu \mathrm{g} / \mathrm{ml}$

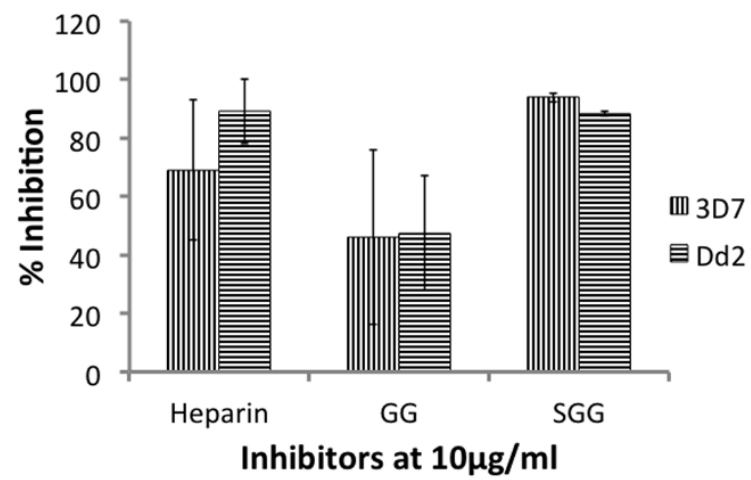

Figure $2 \mid$ In vitro invasion inhibition of $P$. falciparum 3D7 and Dd2. MACS-purified P. falciparum 3D7 and Dd2 schizonts were cultured in 96well plates (ring stage parasitemia $=0 \%$, hematocrit $=1 \%$ ) for $20 \mathrm{~h}$ in the presence of $10 \mu \mathrm{g} / \mathrm{ml}$ heparin, gellan gum (GG), and gellan sulfate (SGG). Ring stage parasitemia were counted using Giemsa stained smears. Percent inhibition of invasion of $P$. falciparum 3D7 and Dd2 are shown. Error bars represent standard deviations from the means of duplicate assays from two independent experiments. 
(a)

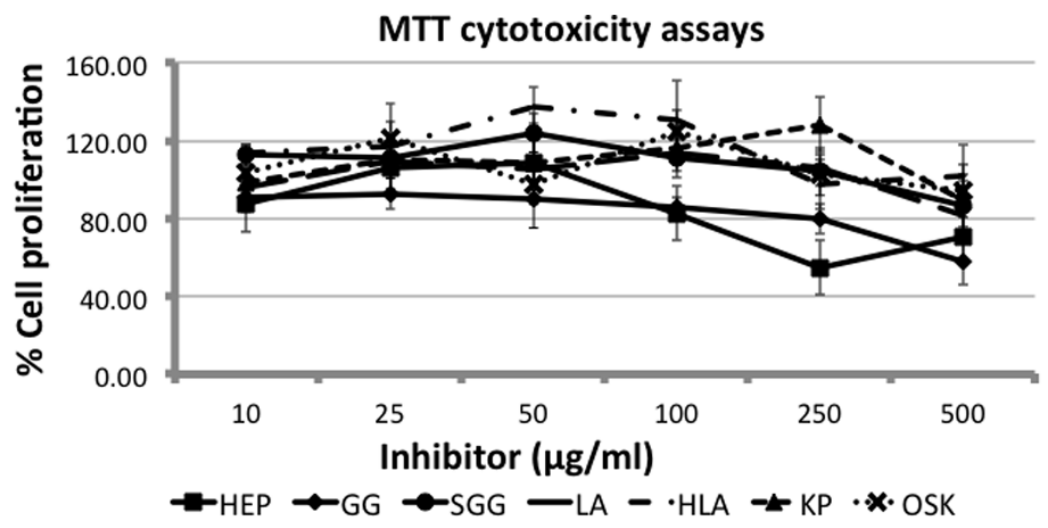

(b)

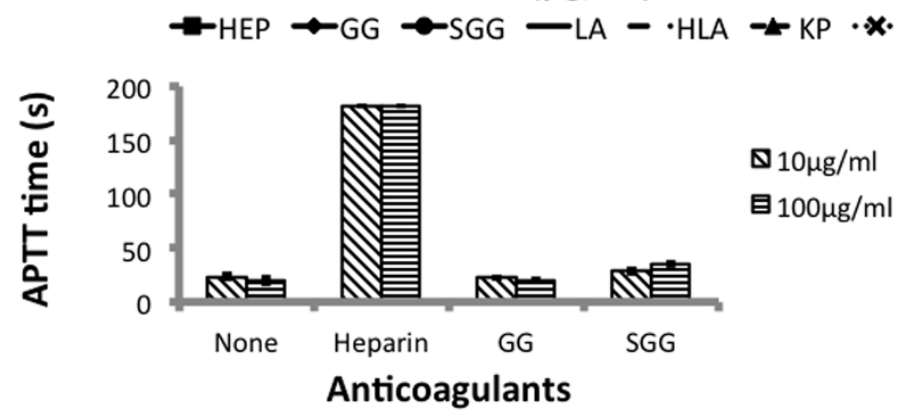

Figure 3 Cytotoxicity and Activated Partial Thromboplastin Time (APTT) assays (a) Cytotoxicity assays using 293T cells. Cultures of 293T cells $(100 \mu \mathrm{l})$ were seeded into 96 -well flat-bottom plates and incubated at $37^{\circ} \mathrm{C}$. At about $50 \%$ confluence, the old media were replaced with fresh media containing the inhibitors heparin (HEP), gellan gum (GG), gellan sulfate (SGG), $\lambda$-carrageenan (LA), hydrolyzed $\lambda$-carrageenan (HLA), $\kappa$-carrageenan $(\mathrm{KP})$, or oversulfated $\kappa$-carrageenan (OSK) and the cells were incubated at $37^{\circ} \mathrm{C}$ for $48 \mathrm{~h}$. MTT $(10 \mu \mathrm{l})$ was then added to the cultures, which were kept in the dark overnight (about $12 \mathrm{~h}$ ) at room temperature. Absorbance was read at $595 \mathrm{~nm}$ and the $\%$ cell viability was computed as: $\%$ cell viability = (absorbance $595 \mathrm{~nm}$ of treated group $\div$ absorbance $595 \mathrm{~nm}$ of control group) $\times 100 \%$. Means and standard deviations from the means were computed from triplicates assays in three independent experiments. (b) APTT assays. Pooled plasma from adult C57BL/6 and BALB/c mice were used for the APTT anticoagulant assays. $10 \mu \mathrm{l}$ of heparin, gellan gum, and gellan sulfate each diluted in PBS were mixed with $100 \mu$ lof plasma for final concentrations of 10 and $100 \mu \mathrm{g} / \mathrm{ml}$ and then incubated at $37^{\circ} \mathrm{C}$ for 1 minute. The APTT assays were performed using Drihemato ${ }^{\circledR}$ APTT Test Reagent Card (A\&T Corporation, Japan) according to manufacturer's instructions. Coagulation times (in seconds) were read in a Drihemato ${ }^{\circledR}$ system Coag2V machine. Two independent experiments for each assay were performed in triplicates and means and standard deviations were calculated.

gellan sulfate were $28.7 \mathrm{~s}$ and $35.7 \mathrm{~s}$, respectively (Figure $3 \mathrm{~B}$ ). For plasma with native gellan gum, mean APTT times were $22.5 \mathrm{~s}$ at $10 \mu \mathrm{g} / \mathrm{ml}$, and $19.7 \mathrm{~s}$ at $100 \mu \mathrm{g} / \mathrm{ml}$. These data demonstrate that our synthesized gellan sulfate does not have strong anticoagulant activity.

\section{Discussion}

Polysaccharides from marine sources are currently gaining popularity for their potential medical and therapeutic applications. Seaweedderived polysaccharides such as carrageenans ${ }^{23}$ and fucoidan ${ }^{50}$ have been shown to inhibit the growth and invasion of $P$. falciparum in vitro. However, in in vivo studies, although a decrease in parasitemia may have been observed, there was no significant improvement in the recovery of mice from the illness ${ }^{24,50}$. In addition, $\lambda$-carrageenan was found to increase the permeability of the blood-brain barrier of rats when injected subcutaneously ${ }^{25}$, which could aggravate the disease by adding to the development of cerebral malaria.

Although the exact mechanism of inhibition by these sulfated polysaccharides has yet to be defined, it appears to involve the interactions of the malarial invasion proteins with the sulfated proteoglycans, like heparan sulfate and chondroitin sulfate, that are present on the surface of most cell types including erythrocytes. These interactions have been demonstrated by Boyle et al. ${ }^{18}$ and our group ${ }^{17,19}$, which showed that heparin interacts with the invasion proteins MSP1 and EBA-140.

Naturally occurring polysaccharides have been modified by sulfation and assessed for their anticoagulant, antibacterial, and antiviral activities. Galactomannans from seed extracts were modified by sulfation and shown to have activities against yellow fever virus and dengue 1 virus in vitro and in vivo ${ }^{51}$, and against HIV in vitro ${ }^{52}$. Sulfated konjac glucomannans have also been shown to act against $\mathrm{HIV}^{53}$.

Modification of $\kappa$-carrageenan improves its action against a variety of pathogens. Low molecular weight $\kappa$-carrageenan $(3 \mathrm{kDa})$ and $\kappa$-carrageenan oligosaccharides (KOS), as well as their sulfated derivatives have anti-influenza virus effects both in vitro and in vivo ${ }^{30,31}$. KOS had also enhanced immunostimulatory and antitumor activities $^{26,27}$. In addition, $\kappa$-carrageenan that was covalently bound to $3^{\prime}-$ azido- $3^{\prime}$-deoxythymidine AZT, was found to be active against HIV in vitro ${ }^{32}$.

Gellan sulfate has been prepared for use in various medical applications, for example, as an artificial ligand to remove the extra domain A containing fibronectin $(\operatorname{EDA}(+) \mathrm{FN})$ from the plasma of rheumatoid arthritis patients ${ }^{42}$, and as a novel anticoagulant ${ }^{43}$.

For these reasons, we synthesized oversulfated $\kappa$-carrageenan and gellan sulfate to assess their activities against malaria parasites in vitro. Elemental analysis showed that our synthesized oversulfated $\kappa$-carrageenan and gellan sulfate had sulfate contents of $13.6 \%$ and $9.57 \%$, respectively (Table 1). The degree of substitution values (DS) were calculated as 3.0 for $\kappa$-carrageenan and 3.7 for gellan ${ }^{54,55}$. Note that the maximum DSs for carrageenan and gellan are 4.0 and 10.0, respectively. Thus, sulfation rates (\%) of $\kappa$-carrageenan and gellan sulfate relative to their maximum DSs were calculated as $75 \%$ and $37 \%$.

The synthesized oversulfated $\kappa$-carrageenan and hydrolyzed $\lambda$ carrageenan showed no activity against Plasmodium parasites, which differs from the findings of previously reported studies with modified carrageenans. These differences could be attributed to the different 
modes of invasion and replication of the viruses within host cells compared with that of parasites.

Our results show that our synthesized gellan sulfate effectively inhibited both the growth and invasion of the $P$. falciparum merozoites in vitro unlike the native gellan gum yet similar to heparin. However, unlike heparin, gellan sulfate is a potential artificial ligand for $\operatorname{EDA}(+) \mathrm{FN}$ from the plasma of rheumatoid arthritis patients because it does not bind to proteins such as plasma fibronectin and antithrombin $\mathrm{III}^{42}$. Yet, gellan sulfates with $17.5 \%$ sulfation or greater were found to be potent anticoagulants, like heparin in APTT assays by Miyamoto et $a l^{43}$. In our cytotoxicity and anticoagulant assays, our synthesized gellan gum, with $37 \%$ sulfation was found to have low cytotoxicity and anticoagulant activity. However, at present, we cannot determine the exact reason for these different results. Given that there is insufficient information about the effects of gellan sulfate in vivo, and considering these results, it is suggested that the synthesized gellan sulfate must be used with caution in future in vivo studies. It will also be of interest to explore the mechanism by which gellan sulfate inhibits growth and invasion of red blood cells by the Plasmodium parasite. In future studies, we intend to investigate if gellan sulfate can inhibit parasite adhesion to the red blood cell similar to other glycosaminoglycans, and how the gellan sulfate molecule interacts with the Plasmodium parasite invasion proteins that interact with heparin such as MSP1 and BAEBL.

\section{Methods}

Polysaccharides and sulfation of $\kappa$-carrageenan and gellan gum. The

polysaccharides used in this study were heparin and $\lambda$-carrageenan (Sigma-Aldrich, St. Louis, Mo.), and $\kappa$-carrageenan and gellan gum (WAKO Pure Chemical Ltd. Co., Osaka, Japan). Hydrolyzed $\lambda$-carrageenan, oversulfated $\kappa$ - carrageenan, and gellan sulfate were synthesized as Yuan et al. ${ }^{26}$ with modifications.

$\lambda$-carrageenan $(1 \%(\mathrm{w} / \mathrm{v})$ in distilled water) was hydrolyzed with $0.1 \mathrm{M} \mathrm{HCl}$ to $\mathrm{pH}$ 4.0 for $1 \mathrm{~h}$ at $70^{\circ} \mathrm{C}^{26}$ with stirring. The reaction was neutralized with $\mathrm{NaHCO}_{3}$ and filtered. The supernatant was concentrated by evaporation and desalted by dialysis against distilled water for $48 \mathrm{~h}$ at $4^{\circ} \mathrm{C}$ using a Cell Sep H1 tube (MWCO 1,000, Orange Scientific Inc. Belgium). The retained solution was lyophilized and dried in vacuo for $24 \mathrm{~h}$ at room temperature.

$\kappa$-carrageenan was sulfated by an $\mathrm{SO}_{3}$-pyridine complex, as previously described ${ }^{26}$. Two grams of lyophilized $\kappa$-carrageenan (from a $1 \%(\mathrm{w} / \mathrm{v})$ solution in distilled water) was added to $N, N$-dimethylformamide (DMF) $(84 \mathrm{ml})$ at $60^{\circ} \mathrm{C}$ for $30 \mathrm{~min}$. To this mixture, $16 \mathrm{ml}$ of $\mathrm{SO}_{3}$-DMF complex was added and the mixture was stirred for $4 \mathrm{~h}$ at $70{ }^{\circ} \mathrm{C}$. The $\mathrm{SO}_{3}$-DMF complex was prepared by dropping $20 \mathrm{ml}$ of $\mathrm{ClSO}_{3} \mathrm{H}$ into $100 \mathrm{ml}$ of DMF in an ice water bath. After cooling to room temperature, the mixture was neutralized with saturated $\mathrm{NaHCO}_{3}$ solution, dialyzed against deionized water for 2 days, and the dialysate was freeze-dried to give sulfated $\kappa$-carrageenan. Gellan $(1 \%(\mathrm{w} / \mathrm{v}))$ was treated with $0.1 \mathrm{M} \mathrm{HCl}$ up to $\mathrm{pH}$ 3.0. The acid-treated gellan gum (insoluble fraction) was recovered by filtration and extensively washed until neutralized and then lyophilized. Sulfation was carried out in the same manner as for $\kappa$ carrageenan to give gellan sulfate. The degree of substitution (DS) of the sulfonyl group for $\kappa$-carrageenan and gellan was defined as the molar number of sulfonyl group per di-saccharide and tetra-saccharide units. DS was calculated based on elemental analysis, according to the equation given by Rochas et al. ${ }^{54}$ as follows: DS = (S\%/atomic mass of S)/(C\%/atomic mass of $\mathrm{C} \times$ number of carbons for one unit). Atomic masses of $\mathrm{C}$ and $\mathrm{S}$ are 12 and 32, respectively. The numbers of carbons in one unit of carrageenan and gellan are 12 and 24, respectively. The sulfation rate (\%) was calculated by taking the ratio of the DS of the sulfonyl group to the maximum DS of 4 for $\kappa$-carrageenan and 10 for gellan.

Prior to being added to the cultures, the polysaccharides were dissolved in distilled water to a concentration of $2 \mathrm{mg} / \mathrm{ml}$, and then filtered through a $0.22-\mu \mathrm{m}$ filter and further diluted with appropriate complete media. Polysaccharides that were insoluble at room temperature, namely $\kappa$-carrageenan, $\lambda$-carrageenan, and gellan gum, were dissolved in distilled water at $80^{\circ} \mathrm{C}$; heparin, oversulfated $\kappa$-carrageenan, hydrolyzed $\lambda$-carrageenan, and gellan sulfate were dissolved at room temperature.

\section{Analytical methods. ${ }^{13} \mathrm{C}$ NMR spectra were obtained from a JEOL $500 \mathrm{MHz}$} spectrometer operating at $500 \mathrm{MHz}$. The spectra for the oversulfated $\kappa$-carrageenan were recorded at $25^{\circ} \mathrm{C}$, whereas the spectra for the native gellan gum and the gellan sulfate were recorded at $90^{\circ} \mathrm{C}$. Chemical shifts $(\delta$ in ppm) were expressed relative to the resonance of $\mathrm{D}_{2} \mathrm{O}\left({ }^{1} \mathrm{H}\right.$ NMR $)(\delta=4.8)$ and DMSO $\left({ }^{13} \mathrm{C} \mathrm{NMR}\right)(\delta=39.5)$. Samples for NMR analysis were dissolved in DMSO (oversulfated $\kappa$-carrageenan $=60 \mathrm{mg} / \mathrm{ml}$; native gellan gum $=5 \mathrm{mg} / \mathrm{ml}$; gellan sulfate $=20 \mathrm{mg} / \mathrm{ml}$ ) and trace amounts of $\mathrm{D}_{2} \mathrm{O}$ were subsequently added.

The concentration of S was analyzed with an Ion Chromatography System Dionex ICS-1100/1600 (Thermo Scientific Inc., MA USA). The concentration of C was analyzed with a 2400 Series II CHNS/O System (Perkin Elmer Inc., MA, USA). DS was calculated using the formula of Rochas et al. ${ }^{54}$ as described earlier.
In vitro culture of Plasmodium falciparum parasites. The chloroquine-sensitive line Plasmodium falciparum 3D7 and the chloroquine-resistant line, P. falciparum Dd2 parasites were maintained in continuous culture as described by Radfar et al. ${ }^{56}$. In this method, the parasites are grown in human $\mathrm{A}^{+}$red blood cells and maintained in culture medium containing RPMI 1640, 25 mM HEPES, $100 \mu$ M hypoxanthine, $12.5 \mu \mathrm{g} / \mathrm{ml}$ gentamicin sulfate supplemented with $2.5 \%(\mathrm{w} / \mathrm{v})$ Albumax II, and $62.5 \mu \mathrm{g} / \mathrm{ml}$ of $\mathrm{NaHCO}_{3}$. Cultures were kept at $37^{\circ} \mathrm{C}, 5 \% \mathrm{CO}_{2}$, and $5 \% \mathrm{O}_{2}$, with daily medium changes; conditions were maintained at $1 \%$ hematocrit and $1 \%$ parasitemia.

Cell culture. Human embryonic kidney derived $293 \mathrm{~T}$ cells were grown at $37^{\circ} \mathrm{C}, 5 \%$ $\mathrm{CO}_{2}$, and $5 \% \mathrm{O}_{2}$ in culture medium containing DMEM, L-glutamine, penicillinstreptomycin, and $62.5 \mu \mathrm{g} / \mathrm{ml} \mathrm{NaHCO}$, supplemented with $10 \%$ fetal calf serum and passaged every two days at $70 \%-80 \%$ confluency.

Growth inhibition. Growth inhibition assays (GIAs) were performed as previously described $^{57}$. Predominantly ring stage cultures were first synchronized by sorbitol treatment. GIAs were carried out at the next day, when the cultures were mostly in the late trophozoite stage. Infected red blood cells were mixed with fresh Type $\mathrm{A}^{+} \mathrm{RBC}$ to make cultures with $0.30 \%$ parasitemia and $1 \%$ hematocrit. A $25-\mu \mathrm{l}$ aliquot of culture was then transferred into 96-well plates and $2.5 \mu \mathrm{l}$ of the inhibitors was added to each well such that the final concentrations were 9, 4.5, 2.25, and $0.9 \mu \mathrm{g} / \mathrm{ml}$. Three independent experiments were performed and all were done in triplicate; parallel cultures were also maintained. The cultures were incubated at $37^{\circ} \mathrm{C}$, with $5 \% \mathrm{CO}_{2}$ and $5 \% \mathrm{O}_{2}$. At $48 \mathrm{~h}$ post-incubation, $5 \mu \mathrm{l}$ of complete medium was added to each culture. Flow cytometry was used to determine the parasitemia after 90-96 h when the parasites were mostly in the trophozoite-schizont stages.

Flow cytometry. Flow cytometry was used to determine the parasitemia of the cultures $^{57}$. Cultures were mixed with $10 \mu \mathrm{g} / \mathrm{ml}$ ethidium bromide in PBS and left at room temperature for $1 \mathrm{~h}$ in the dark. The cell pellets were then resuspended in $500 \mu \mathrm{l}$ of PBS and transferred into flow cytometry tubes. Flow cytometric analysis was carried out on a FACSCalibur cell analyzer (BD Biosciences) and the data were analyzed with WinMDi ver. 2.9.

Invasion inhibition. For the invasion inhibition assays ${ }^{58,59}$, cultures were purified by using a MACS ${ }^{\circledR}$ (Miltenyl Biotec, Japan) magnetic bead separation column to obtain trophozoite and schizont stage parasites. Purified schizonts were mixed with complete medium to obtain hematocrit of $1 \%$, and fresh $\mathrm{A}^{+}$red blood cells were added for a total parasitemia of $5 \%$ with ring stage parasitemia of almost $0 \%$. Cultures $(100 \mu \mathrm{l})$ were transferred into 96-well plates and the inhibitors heparin, gellan gum, and gellan sulfate were added to a final concentration of $10 \mu \mathrm{g} / \mathrm{ml}$. Cultures were incubated for $20 \mathrm{~h}$ at $37^{\circ} \mathrm{C}$, with $5 \% \mathrm{CO}_{2}$ and $5 \% \mathrm{O}_{2}$. For this, two independent assays were done in duplicates.

Assessment of invasion inhibition. Culture supernatants were aspirated and the cell pellets were smeared and stained with Giemsa staining to determine ring stage parasitemia. Means and standard deviations of ring stage parasitemia were obtained, and the percent inhibition was computed by using the following formula: [Eq.2] $\{$ (Mean\% Ring stage parasitemia of untreated cultures - Mean\% Ring stage parasitemia of inhibitor-treated cultures) $\div$ Mean\% Ring stage parasitemia of untreated cultures\} $\times 100 \%$.

Cytotoxicity assays. MTT assays were performed using $293 \mathrm{~T}$ cells. Cells were seeded in 96-well flat-bottom plate with a culture volume of $100 \mu \mathrm{l}$ at a $2: 1000$ dilution and incubated at $37^{\circ} \mathrm{C}, 5 \% \mathrm{CO}_{2}$, and $5 \% \mathrm{O}_{2}$. After $24 \mathrm{~h}$, at about $50 \%$ confluency, the old media were aspirated and replaced with $100 \mu \mathrm{l}$ of complete medium containing inhibitors at concentrations of $500,250,100,50,25$, and $10 \mu \mathrm{g} / \mathrm{ml}$. The cells and inhibitors were then incubated at $37^{\circ} \mathrm{C}, 5 \% \mathrm{CO}_{2}$, and $5 \% \mathrm{O}_{2}$ for $48 \mathrm{~h}$. MTT solution $(10 \mu \mathrm{l}$ of $5 \mathrm{mg} / \mathrm{ml}$ ) was added to the culture, which was kept in the dark at room temperature overnight. The following morning, the old media were aspirated and $100 \mu \mathrm{l}$ of DMSO was added as an MTT solvent. The cells were then assessed by measuring absorbance at $595 \mathrm{~nm}$ and the \% cell viability was computed as follows ${ }^{60}$ : [Eq. 3] \% cell viability $=\left(\right.$ absorbance $595 \mathrm{~nm}$ of treated group $\div$ absorbance $_{595 \mathrm{~nm}}$ of control group) $\times 100 \%$.

In vitro anticoagulant activated partial thromboplastin time (APTT) assay. APTT assays were carried out using plasma from healthy mice ${ }^{49}$. Blood was collected from the hearts of normal adult female C57BL/6 and BALB/c mice under terminal anesthesia. The blood was immediately mixed with $3.2 \%$ trisodium citrate at a $9: 1$ blood: anticoagulant ratio. Plasma was obtained by spinning the blood in a bench top centrifuge for 15 minutes and was then pooled. Heparin, gellan gum, and gellan sulfate $(10 \mu \mathrm{l})$ each diluted in PBS were mixed with $100 \mu \mathrm{l}$ of plasma for a final concentration of 10 and $100 \mu \mathrm{g} / \mathrm{ml}$ and then incubated at $37^{\circ} \mathrm{C}$ for 1 minute. The APTT assays were performed using the Drihemato ${ }^{\circledR}$ APTT Test Reagent Card (A\&T Corporation, Japan) according to manufacturer's instructions. Coagulation times were recorded in seconds (s).

Animals. Adult female C57BL/6 and BALB/c mice purchased from CLEA Japan (Tokyo, Japan) were maintained in controlled light and dark conditions and given food and water ad libitum. 
Ethical statement. The protocol for animal use in experiments was approved by the Committee on the Animal Experiments of the Obihiro University of Agriculture and Veterinary Medicine (Permission No. 25-153, 2013). Animal experiments were conducted in accordance with the Guidelines for Animal Experimentation of the Japanese Association for Laboratory Animal Science and the Fundamental Guidelines for Proper Conduct of Animal Experiment and Related Activities in Academic Research Institutions under the jurisdiction of the Ministry of Education, Culture, Sports, Science and Technology, Japan.

1. World Health Organization. World malaria report: 2012. http://www.who.int/ malaria/publications/world_malaria_report_2012/wmr2012_full_report.pdf (2012). Date accessed: March 27, 2014.

2. Kweka, E. J. et al. Challenges to malaria control and success stories in Africa. Glob. Health Perspect. 1, 71-80 (2013).

3. Sherman, I. W. Malaria: Parasite Biology, Pathogenesis and Protection. (ASM Press, 1998).

4. Yahata, K., Treeck, M., Culleton, R., Gilberger, T. W. \& Kaneko, O. Time-lapse imaging of red blood cell invasion by the rodent malaria parasite Plasmodium yoelii. PLoS One 7, e50780. (2012).

5. Schwartz, L., Brown, G. V., Genton, B. \& Moorthy, V. S. A review of malaria vaccine clinical projects based on the WHO rainbow table. Malar. J. 11, (2012).

6. Cowman, A., Berry, D. \& Baum, J. The cellular and molecular basis for malaria parasite invasion of the human red blood cell. J. Cell Biol 198, 961-971 (2012).

7. Gaur, D. \& Chitnis, C. E. Molecular interactions and signaling mechanisms during erythrocyte invasion by malaria parasites. Curr. Opin. Microbiol. 14, 422-428 (2011).

8. Harvey, K. L., Gilson, P. R. \& Crabb, B. S. A model for the progression of receptorligand interactions during erythrocyte invasion by Plasmodium falciparum. Int. J. Parasitol. 42, 567-573 (2012).

9. Kulane, A. et al. Effect of different fractions of heparin on Plasmodium falciparum merozoite invasion of red blood cells in vitro. Am. J. Trop. Med. Hyg. 46, 589-594 (1992).

10. Clark, D. L., Su, S. \& Davidson, E. A. Saccharide anions as inhibitors of the malaria parasite. Glycoconjugate J. 14, 473-79 (1997).

11. Xiao, L.,Yang, C., Patterson, P. S., Udhayakumar, V. \& Lal, A. A. Sulfated polyanions inhibit invasion of erythrocytes by Plasmodium merozoites and cytoadherence of endothelial cells to parasitized erythrocytes. Infect. Immun. 64, 1373-1378 (1996).

12. Bastos, M. F. et al. Fucosylated chondroitin sulfate inhibits Plasmodium falciparum cytoadhesion and merozoite invasion. (abstract) Antimicrob Agents Chemother doi: 10.1128/AAC.00686-13 AAC.00686-13 (2014)

13. Andrews, K. T., Klatt, N., Adams, Y., Mischnick, P. \& Schwartz-Albiez, R. Inhibition of chondroitin-4-sulfate-specific adhesion of Plasmodium falciparuminfected erythrocytes by sulfated polysaccharides. Infect. Immun. 73, 4288-4294 (2005).

14. Schwartz-Albiez, R. et al. Regioselectively modified sulfated cellulose as prospective drug for treatment of malaria tropica. Glycoconj J 24, 57-65 (2007).

15. Vogt, A. M. et al. Release of sequestered malaria parasites upon injection of a glycosaminoglycan. PLoS Pathog 2, e100. doi:10.1371/journal.ppat.0020100 (2006).

16. Zhang, Y. et al. Proteomic analysis of Plasmodium falciparum schizonts reveals heaparin-binding merozoite proteins. J. Proteome Res. 12, 2185-93 (2013).

17. Kobayashi, K. et al. Analyses of interactions between heparin and the apical surface proteins of Plsamodium falciparum. Sci. Rep. 3, 3178 (2013).

18. Boyle, M. J., Richards, J. S., Gilson, P. R., Chai, W. \& Beeson, J. G. Interactions with heparin-like molecules during erythrocyte invasion by Plasmodium falciparum merozoites. Blood 115, 4559-4568 (2010).

19. Kobayashi, K. et al. Plasmodium falciparum BAEBL binds to heparan sulfate proteoglycans on the human erythrocyte surface. J. Biol. Chem. 285, 1716-1725 (2010).

20. Wilson, D. W., Langer, C., Goodman, C. D., McFadden, G. I. \& Beeson, J. G. Defining the timing of action of antimalarial drugs against Plasmodium falciparum. Antimicrob. Agents Ch. 57, 1455-1467 (2013).

21. Lobo, C. A., Rodriguez, M., Reid, M. \& Lustigman, S. Glycophorin C is the receptor for the Plasmodium falciparum erythrocyte binding ligand PfEBP-2 (baebl). Blood 101, 4628-4631 (2003).

22. WHO. Guidelines for the Treatment of Malaria - 2nd edition (WHO Press, Geneva, 2010).

23. Adams, Y., Smith, S. L., Schwartz-Albiez, R. \& Andrews, K. T. Carrageenans inhibit the in vitro growth of Plasmodium falciparum and cytoadhesion to CD36. Parasitol. Res. 97, 290-294 (2005).

24. James, M. A. \& Alger, N. E. Plasmodium berghei:effect of carrageenan on the course of infection in the A/J mouse. Int. J. Parasitol. 11, 217-220 (1981).

25. Huber, J. D. et al. Blood-brain barrier tight junctions are altered during a $72-\mathrm{h}$ exposure to $\lambda$-carrageenan-induced inflammatory pain. Am. J. Physiol. Heart Circ. Physiol. 283, H1531-H1537 (2002).

26. Yuan, H. et al. Preparation and in vitro antioxidant activity of $\kappa$-carrageenan oligosaccharides and their oversulfated, acetylated, and phosphorylated derivatives. Carbohydr. Res. 340, 685-692 (2005)

27. Yuan, H., Song, J., Li, X., Li, N. \& Dai, J. Immunomodulation and antitumor activity of $\kappa$-carrageenan oligosaccharides. Cancer Lett. 243, 228-234 (2006).
28. Yuan, H., Song, J., Li, X., Li, N. \& Liu, S. Enhanced immunostimulatory and antitumor activity of diferent derivatives of $\kappa$-carrageenan oligosaccharides from Kappaphycus striatum. J. Appl. Phycol. 23, 59-65 (2011).

29. Wang, F. F. et al. Antibacterial activities of kappa-carrageenan oligosaccharides (abstract). Mechanical Engineering and Materials Science 108, 194-199 (2011).

30. Wang, W. et al. In vitro inhibitory effect of carrageenan oligosaccharide on influenza A H1N1 virus. Antiviral Res. 92, 237-246 (2011).

31. Tang, F., Chen, F. \& Li, F. Preparation and potential in vivo anti-influenza virus activity of low molecular-weight $\kappa$-carrageenans and their derivatives. Journal of Appl. Polymer Sci. 127, 2110-2115 (2013).

32. Vlieghe, P. et al. Synthesis of new covalently bound $\kappa$-carrageenan-AZT conjugates with improved anti-HIV activities. J. Med. Chem. 45, 1275-1283 (2002).

33. Ogaji, I. J., Nep, E. I. \& Audu-Peter, J. D. Advances in natural polymers as pharmaceutical excipients. Pharmaceutica Analytica Acta 3, 146 (2012).

34. Rinaudo, M. \& Milas, M. Novel Macromolecules in Food Systems - Developments in Food Science 41 (Elsevier Science B.V., Amsterdam:2000).

35. Shah, J. N., Jani, G. K. \& Parikh, J. R. Gellan gum and its applications - a review. Pharmaceutical Information, Articles and Blogs 5, http://www.pharmainfo.net/ reviews/gellan-gum-and-its-application---review (2007). Accessed June 10, 2013.

36. Goncalves, V. M. F. et al. Structural analysis of gellans produced by Sphingomonas elodea strains by electrospray tandem mass spectrometry. Carbohydr. Polym. 77, 10-19 (2009).

37. Lee, M. W., Tasi, H. F., Wen, S. M. \& Huang, C. H. Photocrosslinkable gellan gum film as an anti-adhesion barrier. Carbohydr Polym 90, 1132-8 (2012).

38. Chang, S. J. et al. Gellan gum films for effective gided bone regeneration. J Med Biol Eng 30, 99-103 (2010).

39. Kim, M. Y., Lee, C. M., Kim, J. N., Cho, K. O. \& Lee, K. Y. Prevention of postsurgical peritoneal adhesion in rats using curdlan and gellan gum hydrogels. Macromol Res doi: 10.1007/s13233-012-0184-1 (2012).

40. Silva-Correira, J. et al. Gellan gum-based hydrogels for intervertebral disc tissueengineering applications. J Tissue Eng Regen Med 5, e97-107 (2011).

41. Oliveira, J. T. et al. Gellan gum injectable hydrogels for cartilage tissue engineering applications: in vitro studies and preliminary in vivo evaluation. (abstract) Tissue Eng Part A 16, 343-53 (2010).

42. Miyamoto, K. et al. Preparation of gellan sulfate as an artificial ligand for removal of extra domain A containing fibronectin. Int. J. of Biol. Macromol. 28, 381-385 (2001).

43. Miyamoto, K. et al. Gellan sulfate selectively suppresses the activation of hemocoagulation factors XI and XII. Mater. Sci. Eng. C 30, 364-368 (2010).

44. Jay, A. J. et al. Analysis of structure and function of gellans with different substitution patterns. Carbohydr. Polym. 35, 179-188 (1998).

45. van de Velde, F. et al. $\mathrm{H}-1$ and c-13 high resolution nmr spectroscopy of carrageenans: Application in research and industry. Trends Food Sci. Tech. 13, 73(2002).

46. van de Velde, F., Pereira, L. \& Rollema, H. S. The revised nmr chemical shift data of carrageenans. Carbohydr. Res. 339, 2309-2313 (2004).

47. de Araujo, C. A. et al. Selective sulfation of carrageenans and the influence of sulfate regiochemistry on anticoagulant properties. Carbohydr. Polym. 91, 483-491 (2013)

48. Thahn, T. T. T., Yasunaga, H., Takano, R., Urakawa, H. \& Kajiwara, K. Molecular characteristics and gelling properties of carrageenan family - 2. Tri-sulfated and tetra-sulfated carrageenans. Polym. Bull. 47, 305-312 (2001).

49. Shih, S., Wang, Z., Guo, S. \& Li, L. Anticoagulant activity of cellulose sulfates with different intrinsic viscosities. Asian J. Pharmaceut. Sci. 2, 38-43 (2007).

50. Chen, J. H., Lim, J. D., Sohn, E. H., Cho, Y. S. \& Han, E. T. Growth-inhibitory effect of a fucoidan from brown seaweed Undaria pinnatifida on Plasmodium parasites. Parasitol. Res. 104, 245-250 (2009).

51. Ono, L. et al. In vitro and in vivo antiviral properties of sulfated galactomannans against yellow fever virus (BeH111 strain) and dengue 1 virus (Hawaii strain). Antiviral Res. 60, 201-208 (2003).

52. Muschin, T., Kanamoto, T., Nakashima, H. \& Yoshida, T. Synthesis and potent biological activities of sulfated galactomannans. Poster presentation: The $9^{\text {th }}$ SPSJ International Polymer Conference (IPC2012) (2012).

53. Bo, S., Muschin, T., Kanamoto, T., Nakashima, H. \& Yoshida, T. Sulfation and biological activities of konjac glucomannan. Carbohydr. Polym. 94, 899-903 (2013).

54. Rochas, C., Lahaye, M. \& Yaphe, W. Sulfate content of carrageenan and agar determined by infrared spectroscopy. Botanica Marina 29, 335-340 (1986).

55. Melo, M. R. S., Feitosa, J. P. A., Freitas, A. L. P. \& dePaula, R. C. M. Isolation and characterization of soluble sulfated polysaccharide from Gracilaria cornea. Carbohydr. Polym. 49, 491-498 (2002).

56. Radfar, A. et al. Synchronous culture of Plasmodium falciparum at high parasitemia levels. Nat. Protoc. 4, 1899-1915 (2009).

57. Persson, K. E. M., Lee, C. T., Marsh, K. \& Beeson, J. G. Development and optimization of high-throughput method to measure Plasmodium falciparumspecific growth inhibitory antibodies. J. Clin. Microbiol. 44, 1665-1673 (2006).

58. Bates, A. H., Mu, J., Jiang, H., Fairhurst, R. M. \& Su, X. Use of magnetically purified Plasmodium falciparum parasites improves the accuracy of erythrocyte invasion assays. Exp. Parasitol. 126, 278-280. (2010). 
59. Spadafora, C., Gerena, L. \& Kopydlowski, K. M. Comparison of the in vitro invasive capabilities of Plasmodium falciparum schizonts isolated by Percoll gradient or using magnetic beads separation. Malar. J. 10, (2011).

60. Edmondson, J. M., Armstrong, L. S. \& Martinez, A. O. A rapid and simple MTTbased spectrophotometric assay for determining drug sensitivity in monolayer cultures. J. Tissue Cult. Meth. 11, 15-17 (1988).

\section{Acknowledgments}

This study was supported by Grants-in-Aids for Young Scientists, and Scientific Research on Innovative Areas (3308) from the Ministry of Education, Culture, Science, Sports, and Technology (MEXT) and for Research on global health issues from the Ministry of Health, Labour and Welfare of Japan, the Bio-oriented Technology Research Advancement Institution (BRAIN), The Naito Foundation, and the Program to Disseminate Tenure Tracking System from the Japan Science and Technology Agency (IST).

We would also like to thank Dr. Mizuki Tomihari and Dr. Kazuro Miyahara of the Veterinary Medical Center of the Obihiro University of Agriculture and Veterinary Medicine for providing resources and assistance in the conduct of the APTT anticoagulation assays.

\section{Author contributions}

F.C.R., K.K. and K.K. designed the experiments. F.C.R. performed the malaria and biological experiments and wrote the main manuscript. A.Ishiwa., Y.E.R. and N.G.V.F. prepared, analyzed and co-wrote the methods and discussion on the synthetic polysaccharides. T.S., H.T., T.I., F.M., H.G., A.I., T.H. and T.I. contributed to the data analysis and discussion. K.Kato edited the manuscript and supervised the study.

\section{Additional information}

Supplementary information accompanies this paper at http://www.nature.com/ scientificreports

Competing financial interests: The authors declare no competing financial interests.

How to cite this article: Recuenco, F.C. et al. Gellan sulfate inhibits Plasmodium falciparum growth and invasion of red blood cells in vitro. Sci. Rep. 4, 4723; DOI:10.1038/srep04723 (2014)

This work is licensed under a Creative Commons Attribution-NonCommercialNoDerivs 3.0 Unported License. The images in this article are included in the article's Creative Commons license, unless indicated otherwise in the image credit; if the image is not included under the Creative Commons license, users will need to obtain permission from the license holder in order to reproduce the image. To view a copy of this license, visit http://creativecommons.org/licenses/by-nc-nd/3.0/ 